\title{
Premature expression of a muscle fibrosis axis in chronic HIV infection
}

\author{
Rebecca L Kusko ${ }^{1}$, Camellia Banerjee ${ }^{1}$, Kimberly K Long ${ }^{1}$, Ariana Darcy ${ }^{1}$, Jeffrey Otis ${ }^{2}$, Paola Sebastiani ${ }^{3}$, \\ Simon Melov ${ }^{4}$, Mark Tarnopolsky ${ }^{5}$, Shalender Bhasin ${ }^{1}$ and Monty Montano ${ }^{1 *}$
}

\begin{abstract}
Background: Despite the success of highly active antiretroviral therapy (HAART), HIV infected individuals remain at increased risk for frailty and declines in physical function that are more often observed in older uninfected individuals. This may reflect premature or accelerated muscle aging.

Methods: Skeletal muscle gene expression profiles were evaluated in three uninfected independent microarray datasets including young (19 to 29 years old), middle aged (40 to 45 years old) and older (65 to 85 years old) subjects, and a muscle dataset from HIV infected subjects (36 to 51 years old). Using Bayesian analysis, a ten gene muscle aging signature was identified that distinguished young from old uninfected muscle and included the senescence and cell cycle arrest gene p21/Cip1 (CDKN1A). This ten gene signature was then evaluated in muscle specimens from a cohort of middle aged (30 to 55 years old) HIV infected individuals. Expression of p21/Cip1 and related pathways were validated and further analyzed in a rodent model for HIV infection.
\end{abstract}

Results: We identify and replicate the expression of a set of muscle aging genes that were prematurely expressed in HIV infected, but not uninfected, middle aged subjects. We validated select genes in a rodent model of chronic HIV infection. Because the signature included p21/Cip1, a cell cycle arrest gene previously associated with muscle aging and fibrosis, we explored pathways related to senescence and fibrosis. In addition to p21/Cip1, we observed HIV associated upregulation of the senescence factor p16INK4a (CDKN2A) and fibrosis associated TGF $\beta 1$, CTGF, COL1A1 and COL1A2. Fibrosis in muscle tissue was quantified based on collagen deposition and confirmed to be elevated in association with infection status. Fiber type composition was also measured and displayed a significant increase in slow twitch fibers associated with infection.

Conclusions: The expression of genes associated with a muscle aging signature is prematurely upregulated in HIV infection, with a prominent role for fibrotic pathways. Based on these data, therapeutic interventions that promote muscle function and attenuate pro-fibrotic gene expression should be considered in future studies.

Keywords: Skeletal muscle, Aging, Gene expression, HIV infection, Senescence

\section{Background}

Despite the considerable success of highly active antiretroviral therapy (HAART), individuals chronically infected with HIV remain at higher risk for declines in musculoskeletal function and increased frailty [1-3], a phenotype also observed in rodent models for HIV infection $[4,5]$. In humans, this functional decline resembles an aging phenotype, since many of these risk factors

\footnotetext{
*Correspondence: mmontano@bu.edu

'Boston University School of Medicine, 650 Albany St. EBRC 646, Boston, MA02118USA

Full list of author information is available at the end of the article
}

are more often seen in the elderly. However, the molecular and regulatory pathways that underlie this process remain vaguely defined.

Studies on muscle aging in both humans and rodent models have shown increases in collagen deposition and fibrotic tissue in aging muscle [6-10], suggesting a skewing in the balance of muscle and fibroblasts with increasing age. Transforming growth factor $\beta$ (TGF $\beta$ ) is a known key regulator in maintaining the balance of collagen in the extracellular matrix as well as in modulating inflammatory responses $[11,12]$. It is normally expressed in muscle after injury but also has the potential to

\section{Biomed Central}

(c) 2012 Kusko et al.; licensee BioMed Central Ltd. This is an Open Access article distributed under the terms of the Creative Commons Attribution License (http://creativecommons.org/licenses/by/2.0), which permits unrestricted use, distribution, and reproduction in any medium, provided the original work is properly cited. 
induce fibrosis around myofibers (for review see [13]). Injection of TGF $\beta$ for ten days has been shown to induce cachexia and generalized tissue fibrosis in nude mice [14]. Furthermore, Carlson and colleagues have shown an upregulation of TGF $\beta$ and associated pathways in muscle in the aging rodent [15]. Based on these observations of muscle aging in model systems and a similar frailty phenotype in rodent models for infection, we were interested in determining whether premature muscle aging occurs during chronic HIV infection, particularly in the context of successful HAART and whether this phenotype is recapitulated in rodent models for HIV.

To evaluate potential muscle aging within the context of HIV infection, we chose to first evaluate gene expression of muscle specimens (that is, vastus lateralis) in uninfected individuals ranging in age from 19 to 85 using previously published datasets for healthy aging and mild sarcopenia to identify an aging gene signature [16,17]. To assess the possibility of accelerated muscle aging in HIV, we then evaluated muscle gene expression profiles in HIV-infected individuals ranging in age from 30 to 55 years old and in a rodent model for HIV infection and used this to identify associated pathways that might explain the phenotype seen. Senescence, a hallmark of aging, has been defined as an irreversible state of cell cycle arrest [18]. In this study, genes and pathways related to muscle senescence and fibrosis were found to be upregulated and were evaluated further. Here, we describe a role for HIV driven fibrosis in skeletal muscle. This is an understudied potential mediator of functional decline in muscle that could open novel avenues for therapeutic intervention.

\section{Methods}

\section{Ethics statement}

These studies were approved by the Boston University Medical Center Institutional Review Board, and the McMaster University and Hamilton Health Sciences Research Ethics Board. These studies were conducted in accordance with the principles of the Declaration of Helsinki and all subjects gave written informed consent for participation.

\section{Study population \\ HIV-infected inclusion criteria}

This study included HIV-positive men, 30 to 55 years old (average age $=43$ years), who had documented weight loss within the previous six months of between $5 \%$ and $15 \%$ of body weight or an actual body mass index (BMI) at screening of between 17 and 20 (equivalent to $85 \%$ to $95 \%$ of the lower limit of ideal weight), an energy intake in excess of $80 \%$ of the recommended dietary allowance, on stable and potent antiretroviral therapy for at least 12 weeks or not starting antiretroviral therapy in the next four months, CD4 cell count greater than $50 / \mathrm{mm}^{3}$ and HIV copy number less than 10,000 copies/ml (less than 400 in six of nine subjects), and were able and willing to provide informed consent and comply with the protocol. Expression profiles were from biopsies of the vastus lateralis at baseline that were not previously published in a testosterone supplementation study [19]. Arrays were run using Affymetrix HGU133A microarray chips.

\section{GEO datasets}

The online datasets GEO362 [20] and GEO 1428 [16] are expression profiles of muscle tissue from normal human muscle biopsies of the vastus lateralis. GEO362 arrayed young men (age 21 to 27) and older men (age 67 to 75 ). GSE 1428 looked at young men (age 19 to 25) and older men (age 70 to 80 ) who were identified as having mild sarcopenia. The de-identified data were downloaded from the Gene Expression Omnibus (GEO) web repository and were used in our analysis. Arrays for both these studies were run using Affymetrix HGU133A microarray chips.

\section{Supplemental healthy HIV negative dataset}

These subjects were enrolled at McMaster University and Hamilton Health Sciences and were all healthy nonexercising males. None were athletes nor were they on medications known to adversely affect muscle (that is, statins). The age intervals for these subjects were 20 to 25 years old, 40 to 45 years old, and 70 to 75 years old. Muscle biopsies of the vastus lateralis were taken at the same time of day and fasted, as previously described [21] and profiled on Roche NimbleGen Human Gene Expression $12 \times 135 K$ Arrays.

\section{Data processing}

Initial processing

All expression data were globally scaled to a per sample mean of 500. Probes were assigned to be present or absent based on the per sample median. Probes with more than $80 \%$ absent calls were removed from GSE1428 and GSE362. Only probes in GSE362 and GSE1428 with at least one sample intensity above the median for each respective set were considered for analysis using Bayesian Analysis of Differentially Expressed Genes (BADGE) [22]. As a result, a total of 9,872 probes from GSE362 and 11,016 probes from GSE1428 were included in our analysis. Remaining probes were analyzed for differential expression using BADGE [22].

\section{Differential expression}

The GSE362 and GSE1428 data sets were analyzed independently. Our analysis found 26 upregulated and 
36 downregulated genes (relative to average gene expression in the arrays being compared) for GSE362 and 19 upregulated and 65 downregulated genes for GSE1428 using a $0.5 \%$ posterior probability of false positive detection. Ten genes overlapped between the two analyses.

\section{Specificity assessment}

To assess whether the ten gene muscle aging signature reflected a generic muscle disease phenotype, we evaluated our ten gene signature in several other muscle aging diseases such as myositis, amyotrophic lateral sclerosis (ALS), and 48 hour immobilization using PEPR (Public Expression Profile Resource; http://pepr.cnmcresearch.org). Our profile was only significant in 48 hour immobilization, and not any of the other diseases studied (data not shown). To test whether the ten gene muscle aging profile in HIV samples clustered selectively with the expression profiles of older subjects' muscles, we used a Bayesian model based cluster analysis of the ten genes. The cluster method was implemented in the software CAGED (Cluster Analysis of Gene Expression Dynamics) which was also used to generate the unsupervised clustered heatmaps [23].

\section{EASE annotation}

Annotation of our gene lists was performed using the National Center for Biotechnology Information (NCBI) software EASE [24]. EASE is an integrated knowledge database that integrates information from OMIM, Refseq, Unigene, and Gene Ontology to search for overrepresented gene categories in user submitted gene lists [25].

\section{Ex vivo validation and pathway analysis TGF $\beta$ protein measurement}

Muscle homogenates were obtained from the gastrocnemius muscle of the wild type and HIV Tg rodent. Briefly, samples were cleaned of all fat and connective tissue and cut into smaller pieces. Specimens were homogenized in RIPA buffer (25 mM Tris pH7.6, $150 \mathrm{mM} \mathrm{NaCl,} \mathrm{1 \%} \mathrm{NP-}$ $40,1 \%$ sodium deoxycholate, $0.1 \%$ SDS) with complete mini protease inhibitor tablets added (Roche, IN, USA). Homogenates were centrifuged at 21,000 g for $15 \mathrm{~min}-$ utes to pellet insoluble matter. Protein concentrations were determined using the BCA reagent (Pierce, Rockford IL, USA). TGF $\beta$ was measured by ELISA using a Quantikine kit (R\&D Systems, Minneapolis, MN, USA) according to the manufacturer's recommendations and values were normalized to total protein concentration. Three HIV Tg and three wild type rodents were used for these measurements. A Students $t$-test of the data was used to test differential expression with a $P$-value $<0.05$ considered as significant.

\section{RNA quantitative realtime PCR}

RNA was generated from gastrocnemius muscles. Briefly, the gastrocnemius muscles were cleaned of all fat and excess connective tissue, cut into smaller pieces and homogenized in TRIzol reagent (Invitrogen, Carlsbad, CA, USA $\}$ ) then processed for RNA. Phase separation was done using chloroform and centrifugation. RNA was precipitated from the aqueous phase using isopropynol and washed using ethanol. The RNA was then cleaned using the RNeasy Mini Kit (Qiagen Sciences, Valencia, CA, USA) and quantified using a Nanodrop spectrophotometer. Primers for rat GAPDH, CDKN1A, FEZ2 and H3F3B were purchased from Superarray (SABiosciences, Frederick, MD, USA) and analyzed using quantitative realtime PCR detection with SYBR green. For genes associated with fibrosis, COL1A1 (F-GGAATGAAGGGACACAGAGGT, R- GAGCTCCATTTTCACCAGGA), COL1A2 (F-GAGCTCCATTTTCACCAGGA, R- CAGCAGCTCCACTCTCACCT), CTGF (F-ATGCTGTGAGGAGTGGGTGT, R- GGCCAAATGTGTCTTCCAGT) and GAPDH (F- ATGACTCTACCCACGGCAAG, R- GGAAGATGGTGATGGGTTTC), primers were used - a kind gift from Dr. Maria Trojanowska (Boston University, MA). They were analyzed using quantitative realtime PCR detection using SYBR green. TaqMan probes for rat p16INK4a, MT1A, MLF1, TPPP3 (also known as CGI-38), MYH8, PDHA1 and GAPDH were purchased from Applied Biosystems (Life Technologies, Carlsbad, CA, USA). Both sets were analyzed using an ABI Prism 7000 Sequence Detection System (Applied Biosystems, Life Technologies, CA, USA). Samples were confirmed to have no DNA contamination by using a realtime PCR reaction without reverse transcriptase. Amplification results were normalized to GAPDH using the $\Delta \Delta \mathrm{Ct}$ method. A Students $t$-test of the data was used to test differential expression with a $P$-value $<0.05$ considered as significant.

\section{Collagen staining and fibrotic index calculation}

Gastrocnemius muscle was flash frozen in liquid nitrogen and later embedded in paraffin. Sections of tissue were processed at $5 \mu \mathrm{m}$ thickness. Tissue was stained using Picrosirius Red (Sigma-Aldrich, St. Louis, MO, USA \}) and Fast Green (Fisher Scientific, Hampton, NH, USA) to look for collagen deposition in the extracellular space. The images for analysis of fibrotic index were taken using Olympus BX41 microscope (Olympus, Center Valley, PA, USA) using DP Controller (Version 3.2.1.276) and DP Manager (Version 3,1,1,208). Bright field images were exposed for $1 / 1500$ seconds. The fibrotic index was calculated as percent area of collagen of the total tissue area using NIH Image J Software (http://rsbweb.nih.gov/ij/). The calculation for fibrotic index is based on the algorithm described in [26]. Six HIV Tg and three wild type rodent muscles were 
quantified after staining. A Students $t$-test of the data was used to test differential expression with a $P$-value $<$ 0.05 considered as significant.

\section{Myosin heavy chain isoform quantification}

Gastrocnemius muscles were embedded in OCT and immediately frozen in isopentane cooled in liquid nitrogen. Serial sections from the mid-belly of the gastrocnemius were cut at $8 \mu \mathrm{m}$ and processed for immunohistochemical detection of slow or fast $\mathrm{MHC}$ protein expression using the ABC method (Vector Labs, Burlingame, CA, USA). Sections were visualized with a Leica microscope and measured using ImageJ software $(\mathrm{NIH}$, Bethesda, MD, USA). Approximately 200 fibers per muscle were analyzed. Data are expressed as the percentage of slow (type I) and fast (type II) MHC types relative to the total pool of MHC isoforms. Four HIV Tg and four wild type rodent muscles were quantified. A Students $t$-test was used to test differential expression with a $P$-value of less than 0.05 considered as significant.

\section{Results and discussion}

Identification of common genes that change expression with muscle aging

To profile muscle in healthy aging, we identified a shared gene expression pattern in healthy and mildly sarcopenic individuals using previously published expression data obtained from the same tissue and microarray platform (that is, Affymetrix HG-U133A). We then evaluated this expression pattern with microarray data from our HIV muscle specimens using the same microarray platform (that is, Affymetrix HG-U133A) as well as with a distinct microarray dataset of young, middle aged and older men using a NimbleGen platform (Table 1). A Bayesian modeling approach was applied, implemented in BADGE [22,23], using muscle expression datasets obtained from the vastus lateralis in GEO series 362 (a dataset including seven young healthy men ages 21 to 27 and eight older men ages 67 to 75 [20]) and GEO series 1428 (a dataset including ten young healthy men ages 19 to 25 and twelve older men with mild sarcopenia ages 70 to 80 [16]). The initial analysis identified 62 age-associated probes that were differentially expressed in the dataset GSE362 and 85 age-associated probes differentially expressed in the dataset
GSE1428. Ten genes were common to both expression sets and are shown for the two datasets as heatmaps in Figures $1 \mathrm{~A}$ and B. Notably the mild sarcopenia in the GEO 1428 series displayed a larger number of differentially expressed genes compared to healthy subjects (data not shown). This ten gene signature was evaluated in another supplemental healthy male dataset run on a NimbleGen microarray platform, comparing subjects 20 to 25 years old with subjects 70 to 75 years old. Most genes (the top six of ten in the heatmap) in the signature were recapitulated in the third set (Figure 1C). The composition of the ten genes shown in all three datasets is as follows: CDKN1A (p21/Cip1), FEZ2, H3F3B DAAM2, MFL1, PDHA1 MT1F, MYH8, CRIM1, and CGI-38.

The GSE362 and GSE1428 genome-wide expression data have been previously published and are available online $[16,20]$. The HIV baseline muscle profiles and the supplemental healthy muscle expression profiles have not been previously published. Cells $/ \mathrm{CMM}=$ cells per cubic milliliter.

\section{Muscle gene expression of middle-aged HIV-infected men resembles older uninfected samples}

Using the muscle aging profile common to both datasets, we evaluated muscle expression profiles from HIVinfected individuals using the same tissue source (vastus lateralis) and microarray platform (Affymetrix HGU133A). The samples are described in [19]. Muscle profiles from these subjects clustered with the older samples in both GSE362 (shown in Figure 2A) and GSE1428 (data not shown) using CAGED [23] cluster analysis. Notably, the clustering was not influenced by viral load, extent of weight loss or CD4 levels (data not shown). Collectively, these data suggest that muscle derived from $\mathrm{HIV}+$ men, on average in their 40s, more closely resembles muscle profiles from individuals in their 70 s consistent with our premise of accelerated muscle aging.

\section{Muscle gene expression of age-matched healthy HIV negative men do not resemble older samples}

We evaluated muscle profiles from young, middle aged and older uninfected men, all derived using the same tissue site and microarray platform (Table 1), to determine whether age, rather than HIV, might account for the observed clustering in Figure 2A. However, CAGED

Table 1 Description of datasets used in this study

\begin{tabular}{|c|c|c|c|c|c|}
\hline Dataset & Age(yrs) & Sample Size(all male) & Tissue source & Array & Condition \\
\hline GSE362 [20] & $21-2767-75$ & $N=7 N=8$ & Vastus lateralis & HG-U133A & Healthy \\
\hline GSE1428 [16] & $19-2570-80$ & $N=10 N=12$ & Vastus lateralis & HG-U133A & Older with sarcopenia \\
\hline $\mathrm{HIV}+[19]$ & $36-51$ & $N=9$ & Vastus lateralis & HG-U133A & $\begin{array}{l}\text { Median VL = } 400 \text { copies/ML } \\
(400-56,844) \text { Median CD4 = } 362 \text { cells/CMM (61-765) }\end{array}$ \\
\hline Supplemental Healthy & $20-2540-4570-75$ & $\mathrm{~N}=10 \mathrm{~N}=10 \mathrm{~N}=10$ & Vastus lateralis & NimbleGen & Healthy \\
\hline
\end{tabular}


A

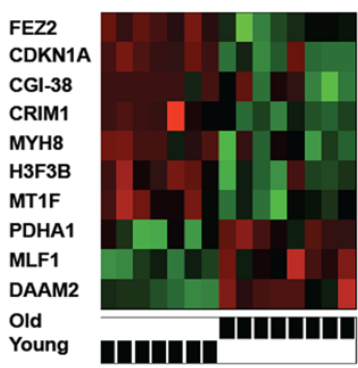

B

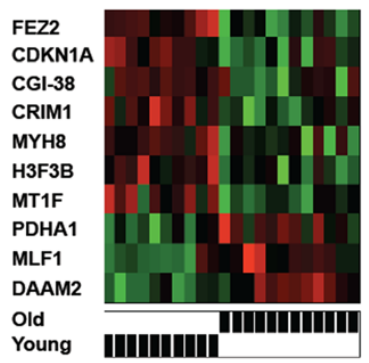

\section{C}

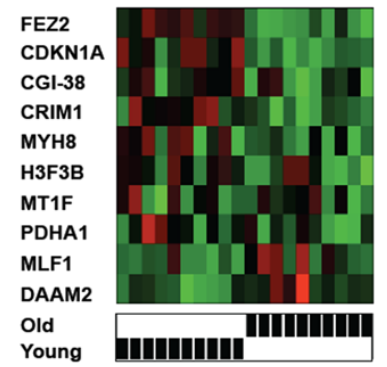

Figure 1 Muscle age profiling. Heatmaps displaying the relative expression of the ten gene muscle aging profile are shown comparing young males to older males in a supervised manner using Affymetrix U133A microarray gene set data for GSE362 (A) and GSE1428 (B) and in a healthy aging dataset using a NimbleGen microarray data $(\mathbf{C})$. The gene expression of the profile is displayed in a supervised manner where intensity reflects relative expression (green = higher, red=lower). Heatmaps were generated using the Heatplus package in the statistical software $\mathrm{R}$ 2.14.0.

cluster analysis of all three groups showed the middle aged men clustering randomly with the young group and the old group, see Figure 2B. Notably, when middle aged healthy men were removed from the analysis, we again observed clear partitioning of the young from the old (Figure 1C), confirming that young and old in this dataset also recapitulate the ten gene muscle signature, as observed in Figure $1 \mathrm{~A}$ and $\mathrm{B}$. These data are consistent with the possibility that HIV infection promotes premature expression of this gene signature in muscle.

Expression of the aging signature in a transgenic rodent model for chronic HIV infection

One of the genes in our signature, the cyclin dependent kinase inhibitor (CDKi) p21/Cip1, has been associated

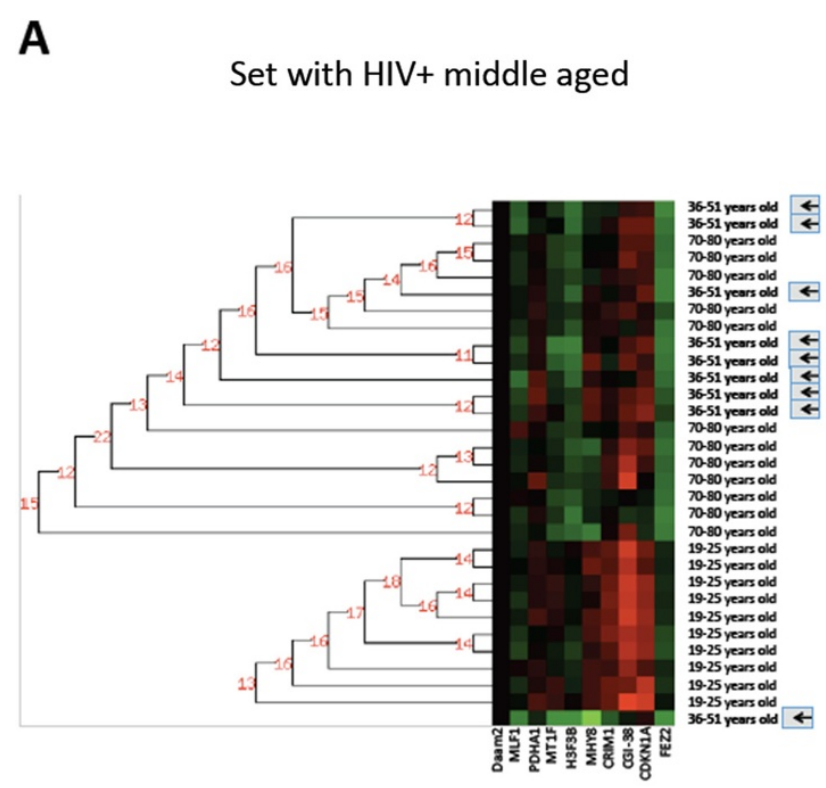

\section{B}

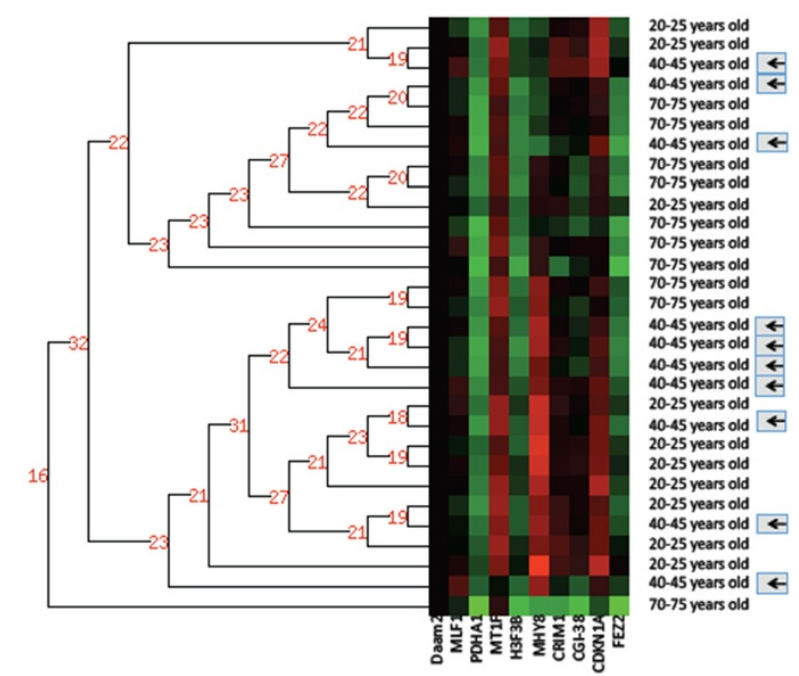

Figure 2 A. Muscle age profiling with HIV. Heatmap displaying the ten gene profile shown in Figure 1, with the HIV group added using GSE1428 (Figure 2A) and GSE362 (similar results, data not shown). The CAGED software was used to cluster samples (shown as rows of the heat map) based on the ten gene muscle aging profile (columns of the heat map). The analysis shows that the young men form a cluster, while HIV samples (designated by arrows and in bold, 36 to 51 years old) cluster with old subjects using the ten gene muscle aging profile. The CAGED cluster analysis uses a model-based procedure that assigns samples to the same cluster if their merging increases the posterior probability of the model. Numbers attached to the branches of the dendrogram represent the posterior odds of the model that merges the branches versus the model that does not. B. CAGED analysis of the expression of the ten gene muscle aging profile is an HIV negative dataset with young (20 to 25 years old), intermediate ( 40 to 45 years old designated by arrows), and old (70 to 75 years old) subjects (this entire dataset used the microarray NimbleGen platform). The gene expression of the profile is displayed in a supervised manner where intensity reflects relative expression (green = higher than average, red = lower than average). CAGED, Cluster Analysis of Gene Expression Dynamics. 
with muscle aging in rodent models for aging $[6,15]$. We were, therefore, interested in whether a transgenic rodent model for HIV infection (HIV Tg) would display premature elevation of $\mathrm{p} 21 / \mathrm{Cip} 1$ expression as well as the other genes in our signature. The HIV $\mathrm{Tg}$ rodent expresses a transgene consisting of a HIV provirus with a functional deletion of $p o l$ and gag regulated by the viral long terminal repeat. These rodents share many similarities to human HIV infection, compared to other rodent models. Specifically, these rodents express the virus in lymph nodes, spleen, kidney, thymus and immune cells including macrophages, $\mathrm{T}$ cells and $\mathrm{B}$ cells, are antigenic to gp120 and shed gp120 into the peripheral blood stream and have immune suppression compared to wild type animals. Furthermore, by five to nine months of age, these animals develop weight loss, neurological abnormalities, respiratory difficulties and other symptoms of AIDS [27,28]. We chose to use the HIV Tg rodent model for chronic infection because this model displays musculoskeletal decline that includes loss in lean muscle and resorption of bone, both phenotypes observed in human HIV infection [4,5]. As shown in Figure 3A, we observed significant up-regulated expression of p21/Cip1, as well as most of the other aging signature genes or gene homologues (for example, Fez2,
H3F3b(H3), CGI-38, MT1, MYH8) using quantitative real time PCR analysis in concordance with expression in the microarray profiles observed in our human muscle specimens. CRIM1 was found to show a trend increase with the HIV Tg compared to the wild type but did not show statistical significance $(P=0.08)$. Notably, three genes, PDHA, DAAM2 and MLF1 were not significantly different between the wild type and HIV transgenic rat (data not shown), possibly indicating species-specific regulation.

\section{p16INK4a is upregulated in a transgenic rodent model for} chronic HIV infection

Recent data have linked elevated p21/Cip1 to other cell cycle arrest genes such as p16INK4a, with healthy aging in muscle stem cells [15] and other tissue specific stem cells $[29,30]$. To assess whether cell cycle arrest was a general feature in the muscle in our rodent model, we measured p16INK4a RNA levels in both the HIV Tg and wild type rodent. Figure $3 \mathrm{~B}$ shows that the levels of p16INK4a were significantly elevated compared with wild type age-matched controls. This further suggests that cell cycle arrest genes are prematurely expressed in muscle during HIV infection, specifically that both the Cip and Ink4 families are induced.
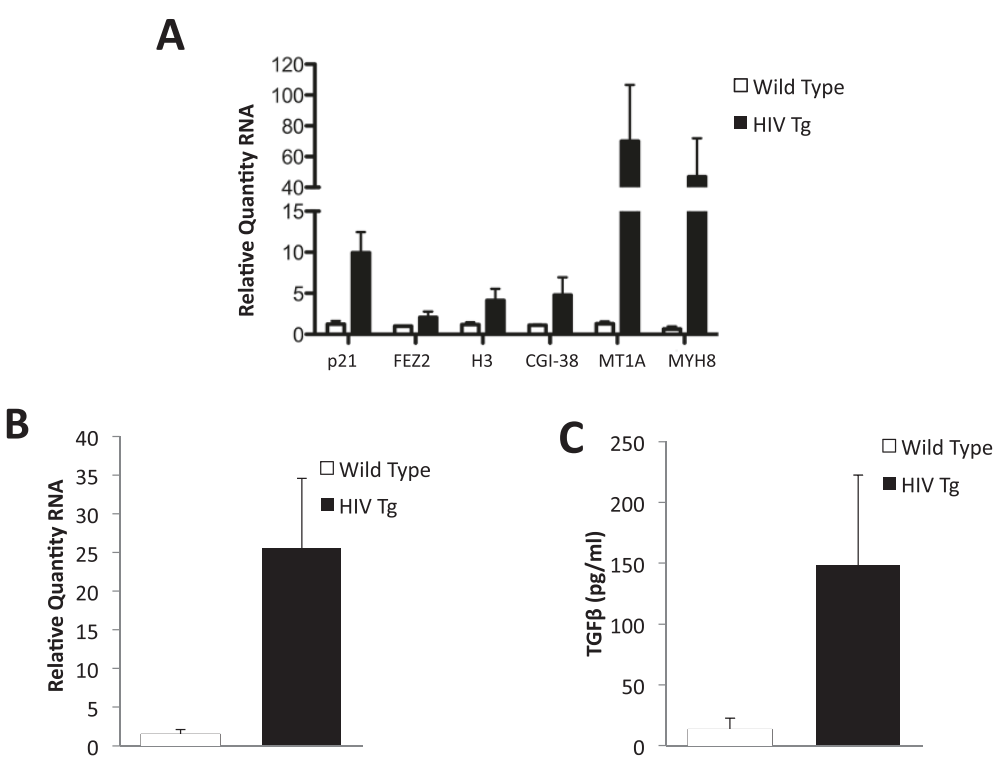

Figure 3 Expression of p21/Cip1, p16INK4a and TGF $\beta$ 1 in the HIV transgenic rodent. A. Realtime RNA PCR validation was observed for p21/Cip1, Fez2, H3, MFL1, MT1F and MYH8 in muscle from HIV transgenic gastrocnemius $(n=4)$ muscle or wild type gastrocnemius muscle $(n=3)$ and shows an increase in genes in HIV Tg compared to age matched wild type controls. All bar plots show mean fold change with error bars indicating the standard deviation. The difference between wild type and HIV Tg is significant to $P \leq 0.05$. B. RT-PCR shows an increase in the cell cycle arrest gene, p16INK4a, in HIV Tg rat $(n=4)$ compared to wild type $(n=3)$. The difference is significant to $P<0.05$. The bar plot indicates mean fold change with the error bars indicative of the standard deviation. C. TGF $\beta 1$ protein levels based on ELISA of muscle homogenates from HIV transgenic gastrocnemius muscle $(n=3)$ or wild type gastrocnemius muscle $(n=3)$ show increased levels in the HIV Tg rodent at a significance of $P<0.05$. Bar plots indicate mean protein levels with error bars indicative of the standard deviation. TGF $\beta$, transforming growth factor $\beta$. 
TGF $\beta$ is upregulated in a transgenic rodent model for chronic HIV infection

The TGF $\beta$ family members, including TGF $\beta$ itself, as well as the growth antagonist myostatin, have been shown to upregulate p21/Cip1 and p16INK4a in aging muscle in both the stem cell population and the muscle fibers in mice [6,15]. Expression of both p21/Cip1 and p16INK4a has also been shown to be upregulated in a number of human aging studies in leukocytes, fibroblasts, neural tissue and pancreatic islet cells [18,29-39]. This upregulation of p16INK4a is thought to be associated with aging and senescence. To evaluate whether TGF $\beta$ protein might also be upregulated in our HIV muscle in addition to the senescence-associated genes, we measured protein levels in muscle homogenates from HIV Tg and age-matched wild type rodents at the onset of bone and muscle loss (approximately seven months of age). As shown in Figure 3C, we observed a dramatic increase in TGF $\beta$ protein levels in HIV Tg animals compared to wild type controls, consistent with elevated TGF $\beta$ in muscle aging studies in rodents [15].

The collagen gene inducer, CTGF, as well as collagen gene expression are induced in a transgenic rodent model for chronic HIV infection

Elevated TGF $\beta$ levels have been previously linked to deposition of collagen and tissue fibrosis of lymph nodes in both human HIV infection and in simian immunodeficiency virus (SIV) models of infection [40,41]. Furthermore, in aging, muscle from rodents has been shown to convert to a fibrogenic phenotype [6]. However, fibrotic genes and collagen deposition in skeletal muscle have not yet been described in the context of HIV infection. We, therefore, measured genes associated with TGF $\beta$ and collagen deposition in the hindlimb muscle (gastrocnemius) of the wild type and HIV Tg rodent. We first examined the collagen transcriptional inducer, connective tissue growth factor (CTGF), a factor that mediates TGF $\beta$ induced collagen gene expression $[42,43]$. As shown in Figure 4A, there was an increase in expression in CTGF in the HIV Tg rodents. We then measured the expression of collagen genes, COL1A1 and COL1A2. As shown in Figure 4B, these genes were also upregulated in the HIV Tg rodent.

\section{The HIV tg rodent muscle exhibits fibrosis and fiber type switching in muscle}

Because we saw evidence of the upregulation of collagen genes in HIV, to evaluate whether collagen deposition was detectable in association with upregulated TGF $\beta$ and collagen genes, we analyzed gastrocnemius tissue sections and quantified collagen content. As shown in Figure 5, there was a significant increase in muscle collagen deposition in the HIV Tg rodent compared to the wild type controls. This is consistent with aging muscle, which also shows an increase in collagen deposition [6]. Since aging is also associated with a shift in fiber types, specifically an increase in slow twitch and a decrease in fast twitch [4446], we analyzed the types of fibers seen in the gastrocnemius in the wild type compared to the HIV Tg. We found that as with aging, there is a significant increase in slow twitch fibers and decrease in the fast twitch fibers in the HIV Tg rat compared to the wild type.

\section{Conclusions}

With the success of anti-retroviral therapy in suppressing viral burden there has been a profound influence on the course of HIV infection from a lethal to a managed
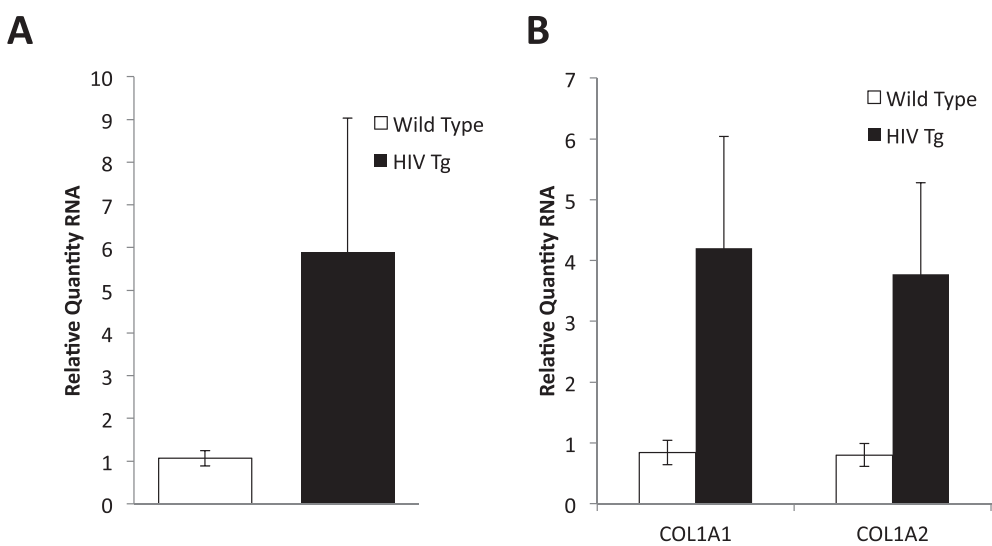

Figure 4 Expression of collagen genes in the HIV transgenic rodent model. A. The collagen transcriptional regulator, CTGF, is increased in HIV Tg rat muscle $(n=3)$ compared to wild type $(n=3)$ using realtime RNA PCR at a significance of $P<0.05$. The bar plots indicate mean fold change with error bars indicative of standard deviation. B. The collagen genes, COL1A1 and COL1A2 are increase in HIV Tg rat muscle ( $n=3$ ) compared to wild type $(n=3)$ using realtime RNA PCR. The bar plots indicate mean fold change with the error bars indicative of standard deviation. The difference between wild type and HIV Tg rodents are significant to $P<0.05$. CTGF, connective tissue growth factor. 


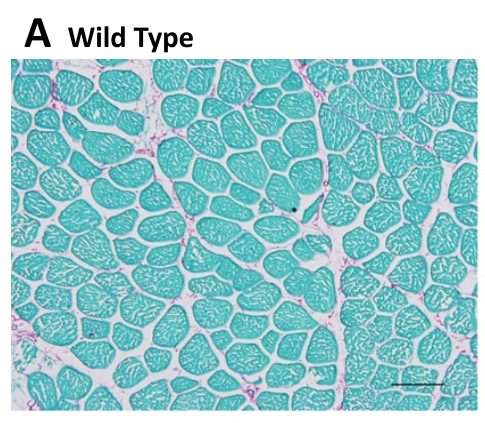

\section{B HIV Tg}

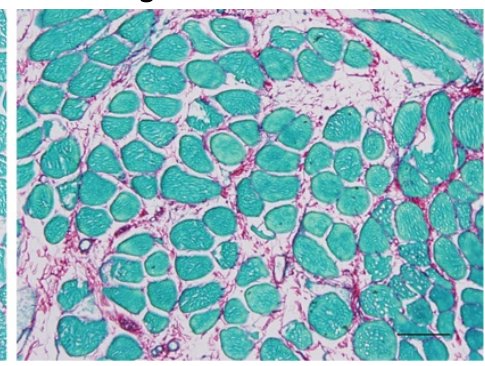

C

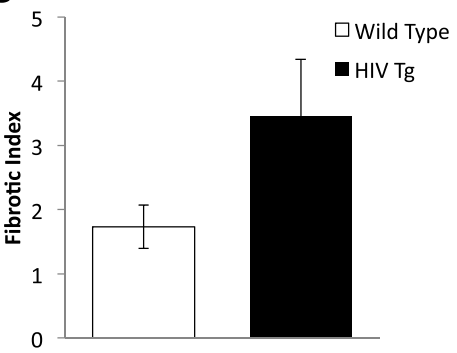

D

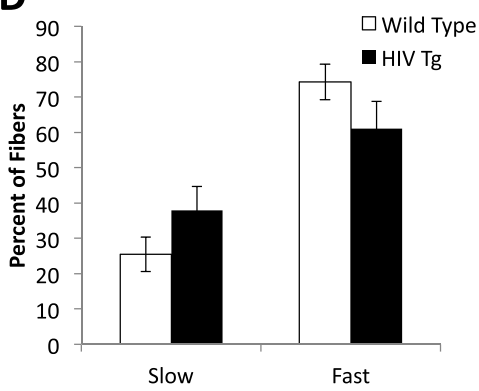

Figure $\mathbf{5}$ Collagen deposition and fiber type switching in the HIV transgenic rat. Histological muscle sections of the gastrocnemius muscle of HIV Tg $(n=6)$ and control wild type rats $(n=3)$ were stained using Picrosirius Red for collagen. Representative images are shown in $\mathbf{A}$ and $\mathbf{B}$. The scale bar equals $200 \mu \mathrm{m}$. A. Tissue section of the gastrocnemius muscle in wild type rat shows collagen deposition in red in intracellular space. B. Tissue section of gastrocnemius muscle from HIV Tg rat shows increased collagen deposition in red in the intracellular spaces. C. Quantification of the area of picrosirius red staining using Image J shows that there is an increase in fibrotic index in the HIV Tg rat compared to the wild type at a significance of $P<0.05$. The bar plot indicates mean percent area of collagen with the error bars indicative of the standard deviation. D. Quantification of fiber type was done using Image J on control wild type $(n=4)$ and HIV Tg $(n=4)$. The bar plot indicates the percentage of fibers of each type with error bars indicative of standard deviation.

chronic disease. Nevertheless, HIV-infected individuals remain at higher than expected risk for a number of complications typically associated with aging [47]. Aging is often defined on the basis of functional capacity, or frailty, rather than the collection of age-associated diseases $[48,49]$. Frailty, as defined in the elderly by Fried and colleagues, [50] has been previously evaluated in men with HIV $[1,2]$ wherein a significant frailty-related phenotype (FRP) has been observed. Margolick and colleagues have also reported the premature occurrence of age-adjusted frailty with HIV infection [1-3].

With the advancing age of the HIV-infected population, the role of aging-associated inflammation in compromising normal tissue remodeling is becoming increasingly more relevant. In 2006, approximately one third of people with AIDS in the US were over age 50 [49]. Chronic inflammation that occurs with increasing age has been shown to adversely affect muscle homeostasis [51-53], with aging muscle increasingly likely to exhibit changes in extracellular matrix resembling a fibrogenic phenotype [6-9]. Recently, SIV infection has been linked with fibroblast deposition of collagen and increased fibrosis in lymph nodes associated with an increase in local TGF $\beta$ production $[40,54,55]$. However, a detailed study of fibrosis in skeletal muscle in chronic HIV infection has not been done to date. In this study, we use an aging signature in muscle to identify and evaluate aging- and fibrosis-related pathways in the rodent model of HIV. We show gene expression changes and phenotypic changes in muscle in an HIV Tg rodent model similar to that seen in aging. We see an upregulation of genes associated with aging muscle prematurely expressed in HIV in both the human and the rat. We, furthermore, show changes in both fiber type composition and in fibrotic collagen deposition in the HIV rodent. Previous work by our laboratory in humans has also shown that an increase in CTGF expression in vastus lateralis from HIV-positive men was downregulated with anabolic supplementation in microarray analysis (see supplemental data in [19]), consistent with our findings of a dysregulation of CTGF in HIV. Collectively these data suggest that the genotypic and phenotypic changes that we see might be related to HIV infection. This data also elucidate pathways HIV might use that result in increased risk for a frailty-related phenotype.

Three independent datasets were used in this study to identify an aging gene signature that differs between older individuals and younger individuals. This signature 
is prematurely expressed in HIV-positive subjects. The aging profile genes include: CDKN1A/p21, FEZ2, H3F3B, DAAM2, MLF1, PDHA1, MT1F, MYH8 and CRIM1. FEZ2 is a factor that interacts with a number of transcription controlling proteins to influence chromatin remodeling and apoptosis [56]. H3F3B is a replacement histone that constitutes the major form of histone $\mathrm{H} 3$ in senescent cells [57]. DAAM2 regulates cell fate and actin-cytoskeleton re-organization through WNT signaling [58]. Previous studies have shown that changes in actin cytoskeleton turnover can trigger an increase in levels of reactive oxygen species (ROS) in the cytosol and, therefore, decrease cellular viability [59]. MLF1 suppresses COP1 through COP9 thereby stabilizing p53 [60]. PDHA1 is a mitochondrial metabolic enzyme that provides the link between glycolysis and the TCA cycle [61]. MT1F and MT1A have a metalloregulatory function in repair, growth, and differentiation [62] with MT1A being a similar family methallothionein to MT1F in the rat. MYH8 is a member of the myosin protein family and is involved in muscle contraction by interacting with actin filaments [63]. CRIM1 is a membrane bound protein that is known to interact with the TGF $\beta$ superfamily [64]. CDKN1A (p21/Cip1) is a well-recognized cell cyclin dependent kinase inhibitor that has been associated with muscle aging and cellular senescence that has not been previously linked with muscle aging in HIV infection. Whether these genes reflect a larger network of regulators in aging that are co-opted in HIV is beyond the scope of this study and will require further network and pathway analysis of these genes and their potential interactions during healthy and dysregulated muscle aging. It is of note that while these genes displayed a robust signature in our two initial datasets, GSE363 and GSE 1428, the signature did not fully recapitulate in a third cohort (shown in Figure 1C). Furthermore, the genes that erode in the heatmap for the third cohort include PDHA, MLF1 and DAAM2 and are the same genes that did not show significant changes in the HIV Tg rat model compared to the wild type (data not shown). This data suggest that these three genes may not remain as robust as the others and will require further analysis of additional independent datasets.

In this study, we see selected senescence-ssociated pathways in addition to the fibrosis pathways prematurely expressed in HIV infection. Based on these results, we propose that HIV infection may represent an uncoupled and dysregulated aging phenotype that increases the risk for muscle fibrosis. Senescence-associated pathways have been previously implicated in muscle aging, but have not been directly linked to HIV infection. Recent studies on senescence and aging indicate that cellular senescence is accompanied by a striking increase in the secretion of 40 to 80 factors $[34,38,65,66]$, termed the 'senescence-associated secretory phenotype' or SASP [35], an ensemble of factors that includes multiple inflammatory cytokines. Previous studies on senescence show that the cells responsible for the release of SASP factors show an upregulation in key senescence-associated genes including p21 and p16INK4a [35-37,39,65]. In muscle, these genes are thought to contribute to a decreased regenerative capacity with aging that has been seen in rodents as they have been detected in both fibers and satellite cells $[15,53]$. We show the premature upregulation of p21/Cip1 in both human and rodent muscle tissue with HIV and the premature upregulation of p16INK4a in rodent muscle tissue suggesting that a senescence axis might be activated early with HIV infection. What cell types in the muscle (that is, satellite cells, fibroblast or mature muscle cells) contribute to the changes in gene expression is beyond the scope of this study and will require further analysis. Furthermore, whether or not the upregulation of these genes contributes to a dysregulation of the satellite cells in HIV muscle leading to an aging phenotype is an intriguing area of future study.

As previously mentioned, many of the factors that increase in serum with aging include inflammatory cytokines including TGF $\beta$, TNF $\alpha$ and IL-6 [34-36,38,39,6769]. Some of these circulating factors have been shown to dampen proliferation of satellite cells, lead to remodeling of the stem cell niche and lead to dysregulation of stem cell function specifically in muscle [15,53,70]. HIV is also associated with an increase in systemic inflammatory burden, especially in serum factors like IL-6 $[1,2,69,71,72]$. Increases in systemic inflammatory cytokines are also known to lead to a loss of muscle mass [73-78]. Whether the gene signature that we see in HIV and aging is due to a common increase in inflammatory burden or whether HIV infection itself drives both the signature and inflammatory process is an intriguing question that remains beyond the scope of this study.

In addition to inflammation, previous studies dissecting acute conditions leading to muscle atrophy have revealed a common role for the ubiquitin proteasome pathway mediated by the E3 ligases MuRF1 and MAFBx to promote muscle protein degradation, notably the muscle structural protein myosin heavy chain [79-82]. Upstream activators for these atrogenes include FOXO and NFKB pathways [83] and have been identified in multiple rodent models of induced wasting, notably cancer-associated cachexia [84] and also HIV in a rodent model for infection [5]. While we do not see an upregulation of atrogenes in our genetic signature in aging, the HIV transgenic rodent model has previously been shown to have an increase in MuRF1 compared to the wild type [4]. Therefore, the atrogene pathway may represent another pathway in addition to the fibrotic and senescence pathways we study that shows dysregulation from the normal aging pattern seen specifically in HIV. 
The cause of HIV-related frailty and muscle loss is an area that has been understudied with the etiology poorly understood. Further evaluation of both the senescent and fibrotic pathways and genes in association with muscle function in healthy aging and in HIV infection should better assist in identifying molecular pathways associated with frailty in both conditions. Increased frailty is likely to create a substantial burden on future health care, underscoring a need for diagnostic biomarkers and early detection in this population. The role of chronic inflammation driven fibrosis in HIV is an understudied potential mediator of functional decline. Further characterization of this phenotype could open avenues for therapeutic intervention to prevent fibrosis associated declines in physical function.

\section{Abbreviations \\ AIDS: Acquired Immunodeficiency Syndrome; ALS: amyotrophic lateral sclerosis; BADGE: Bayesian analysis of differential gene expression; CAGED: cluster analysis of gene expression dynamics; CDKi: cyclin dependent kinase inhibitor; CMM: cubic millimeter; CTGF: connective tissue growth factor; ELISA: enzyme-linked immunoassay; FRP: frailty related phenotype; GEO: gene expression omnibus; HAART: highly active antiretroviral therapy; HIV Tg: HIV transgenic; HIV: human immunodeficiency virus; IL-6: interleukin 6; MAFBx: muscle atrophy F-box; MHC: myosin heavy chain; MuRF1: muscle RING finger protein 1; NCBI: National Center for Biotechnology Information; OCT: optimal cutting temperature medium; OMIM: Online Mendelian Inheritance in Man; PCR: polymerase chain reaction; RNA: ribonucleic acid; ROS: reactive oxygen species; SASP: senescence associated secretory phenotype; SIV: simian immunodeficiency virus; TCA: tricarboxylic acid cycle; TGF $\beta 1$ : transforming growth factor $\beta$ 1; TNFa: tumor necrosis factor $a$; US: United States; WT: wild type.}

\section{Competing interests}

The authors declare that they have no competing interests.

\section{Acknowledgments}

The authors wish to acknowledge the Boston OAIC Pepper Center and the Longevity Consortium for their encouragement and support of this study, and thank M. Trojanowska for the gift of the rodent COL1A1, COL1A2, CTGF and GAPDH primers and P. Haines for technical expertise. This work was supported by U.S. National Institutes of Health grants R01 AR055115 (M.M.) and the Boston OAIC Pepper Center grant 5P30 AG31679 (S.B.) The following reagent was obtained through the AIDS Research and Reference Reagent Program, Division of AIDS, NIAID, NIH: U1/HIV-1 from Dr. Thomas Folks.

\section{Author details}

'Boston University School of Medicine, 650 Albany St. EBRC 646, Boston, MA02118USA. ²Emory University, Atlanta, GA 30322, USA. ${ }^{3}$ Boston University School of Public Health, Boston, MA 02118, USA. ${ }^{4}$ Buck Institute, Novato, CA 94945, USA. ${ }^{5}$ McMaster University, Toronto, ON L8S, Canada.

\section{Authors' contributions}

$B K$ and $C B$ contributed equally to this work. BK, CB and MM wrote the manuscript. KL, CB and AD performed the experiments. JO, SM, MT and SB provided muscle tissue and/or unpublished microarray expression data. BK, PS and MM conceived of the study, coordinated the experimental approach and drafted the manuscript. All authors read and approved the final manuscript.

\section{Received: 8 February 2012 Accepted: 7 June 2012}

Published: 7 June 2012

\section{References}

1. Desquilbet $L$, Jacobson LP, Fried LP, Phair JP, Jamieson BD, Holloway M, Margolick JB: HIV-1 infection is associated with an earlier occurrence of a phenotype related to frailty. J Gerontol A Biol Sci Med Sci 2007, 62:1279-1286
2. Desquilbet L, Margolick JB, Fried LP, Phair JP, Jamieson BD, Holloway M, Jacobson LP: Relationship between a frailty-related phenotype and progressive deterioration of the immune system in HIV-infected men. J Acquir Immune Defic Syndr 2009, 50:299-306.

3. Margolick J, Detels R: Phair JP, Rinaldo C, Jacobson LP: Earlier Occurrence of the Frailty Phenotype in HIV + Men than in HIV- Men: The MACS. In: CROI. Boston; 2011.

4. Otis JS, Ashikhmin Yl, Brown LA, Guidot DM: Effect of HIV-1-related protein expression on cardiac and skeletal muscles from transgenic rats. AIDS Res Ther 2008, 5:8.

5. Vikulina T, Fan X, Yamaguchi M, Roser-Page S, Zayzafoon M, Guidot DM, Ofotokun I, Weitzmann MN: Alterations in the immuno-skeletal interface drive bone destruction in HIV-1 transgenic rats. Proc Natl Acad Sci U S A 2010, 107:13848-13853.

6. Brack AS, Conboy MJ, Roy S, Lee M, Kuo CJ, Keller C, Rando TA: Increased Wnt signaling during aging alters muscle stem cell fate and increases fibrosis. Science 2007, 317:807-810.

7. Grounds MD: Age-associated changes in the response of skeletal muscle cells to exercise and regeneration. Ann N Y Acad Sci 1998, 854:78-91.

8. Schiaffino S, Partridge T: Skeletal Muscle Repair and Regeneration. Dordrecht: Springer; 2008

9. Goldspink G, Fernandes K, Williams PE, Wells DJ: Age-related changes in collagen gene expression in the muscles of mdx dystrophic and normal mice. Neuromuscul Disord 1994, 4:183-191.

10. Sadeh M: Effects of aging on skeletal muscle regeneration. J Neuro/ Sci 1988, 87:67-74.

11. Li MO, Wan YY, Sanjabi S, Robertson AK, Flavell RA: Transforming growth factor-beta regulation of immune responses. Annu Rev Immunol 2006, 24:99-146.

12. Branton MH, Kopp JB: TGF-beta and fibrosis. Microbes Infect 1999, 1:13491365.

13. Wynn TA: Cellular and molecular mechanisms of fibrosis. J Pathol 2008, 214:199-210.

14. Zugmaier G, Paik S, Wilding G, Knabbe C, Bano M, Lupu R, Deschauer B, Simpson S, Dickson RB, Lippman M: Transforming growth factor beta 1 induces cachexia and systemic fibrosis without an antitumor effect in nude mice. Cancer Res 1991, 51:3590-3594.

15. Carlson ME, Hsu M, Conboy IM: Imbalance between pSmad3 and Notch induces CDK inhibitors in old muscle stem cells. Nature 2008, 454:528-532.

16. Giresi PG, Stevenson EJ, Theilhaber J, Koncarevic A, Parkington J, Fielding RA, Kandarian SC: Identification of a molecular signature of sarcopenia. Physiol Genomics 2005, 21:253-263.

17. Welle S, Brooks A, Thornton CA: Senescence-related changes in gene expression in muscle: similarities and differences between mice and men. Physiol Genomics 2001, 5:67-73.

18. Krishnamurthy J, Torrice C, Ramsey MR, Kovalev GI, Al-Regaiey K, Su L, Sharpless NE: Ink4a/Arf expression is a biomarker of aging. J Clin Invest 2004, 114:1299-1307.

19. Montano M, Flanagan JN, Jiang L, Sebastiani P, Rarick M, LeBrasseur NK, Morris CA, Jasuja R, Bhasin S: Transcriptional profiling of testosteroneregulated genes in the skeletal muscle of human immunodeficiency virus-infected men experiencing weight loss. J Clin Endocrinol Metab 2007, 92:2793-2802

20. Welle S, Brooks Al, Delehanty JM, Needler N, Thornton CA: Gene expression profile of aging in human muscle. Physiol Genomics 2003, 14:149-159.

21. Abadi A, Glover El, Isfort RJ, Raha S, Safdar A, Yasuda N, Kaczor JJ, Melov S, Hubbard A, Qu X, Phillips SM, Tarnopolsky M: Limb immobilization induces a coordinate down-regulation of mitochondrial and other metabolic pathways in men and women. PLoS One 2009, 4:e6518.

22. Sebastiani P, Mandl KD, Szolovits P, Kohane IS, Ramoni MF: A Bayesian dynamic model for influenza surveillance. Stat Med 2006, 25:1803-1816. discussion 1817-1825.

23. Ramoni MF, Sebastiani P, Kohane IS: Cluster analysis of gene expression dynamics. Proc Natl Acad Sci U S A 2002, 99:9121-9126.

24. Hosack DA, Dennis G Jr, Sherman BT, Lane HC, Lempicki RA: Identifying biological themes within lists of genes with EASE. Genome Biol 2003, 4: R70.

25. da Huang W, Sherman BT, Lempicki RA: Systematic and integrative analysis of large gene lists using DAVID bioinformatics resources. Nat Protoc 2009, 4:44-57. 
26. Tanano H, Hasegawa T, Kimura T, Sasaki T, Kawahara H, Kubota A, Okada A: Proposal of fibrosis index using image analyzer as a quantitative histological evaluation of liver fibrosis in biliary atresia. Pediatr Surg Int 2003, 19:52-56.

27. Ray PE, Liu XH, Robinson LR, Reid W, Xu L, Owens JW, Jones OD, Denaro F, Davis HG, Bryant JL: A novel HIV-1 transgenic rat model of childhood HIV1-associated nephropathy. Kidney Int 2003, 63:2242-2253.

28. Reid W, Sadowska M, Denaro F, Rao S, Foulke J Jr, Hayes N, Jones O, Doodnauth D, Davis H, Sill A, O'Driscoll P, Huso D, Fouts T, Lewis G, Hill M, Kamin-Lewis R, Wei C, Ray P, Gallo RC, Reitz M, Bryant J: An HIV-1 transgenic rat that develops HIV-related pathology and immunologic dysfunction. Proc Natl Acad Sci U S A 2001, 98:9271-9276.

29. Itahana K, Zou Y, Itahana Y, Martinez JL, Beausejour C, Jacobs JJ, Van Lohuizen M, Band V, Campisi J, Dimri GP: Control of the replicative life span of human fibroblasts by p16 and the polycomb protein Bmi-1. Mol Cell Biol 2003, 23:389-401.

30. Liu Q, Song $L$, Xu WQ, Zhao L, Zheng L, Yan ZW, Fu GH: Expression of cytoplasmic p16 and anion exchanger 1 is associated with the invasion and absence of lymph metastasis in gastric carcinoma. Mol Med Report 2009, 2:169-174

31. Campisi J: Cellular senescence as a tumor-suppressor mechanism. Trends Cell Biol 2001, 11:S27-S31.

32. Krishnamurthy J, Ramsey MR, Ligon KL, Torrice C, Koh A, Bonner-Weir S, Sharpless NE: p16INK4a induces an age-dependent decline in islet regenerative potential. Nature 2006, 443:453-457.

33. Molofsky AV, Slutsky SG, Joseph NM, He S, Pardal R, Krishnamurthy J, Sharpless NE, Morrison SJ: Increasing p16INK4a expression decreases forebrain progenitors and neurogenesis during ageing. Nature 2006, 443:448-452.

34. Coppe JP, Desprez PY, Krtolica A, Campisi J: The senescence-associated secretory phenotype: the dark side of tumor suppression. Annu Rev Pathol 2010, 5:99-118.

35. Coppe JP, Patil CK, Rodier F, Sun Y, Munoz DP, Goldstein J, Nelson PS, Desprez PY, Campisi J: Senescence-associated secretory phenotypes reveal cell-nonautonomous functions of oncogenic RAS and the p53 tumor suppressor. PLoS Biol 2008, 6:2853-2868.

36. Kuilman T, Peeper DS: Senescence-messaging secretome: SMS-ing cellular stress. Nat Rev Cancer 2009, 9:81-94.

37. Beausejour CM, Krtolica A, Galimi F, Narita M, Lowe SW, Yaswen P, Campisi $\mathrm{J}$ : Reversal of human cellular senescence: roles of the p53 and p16 pathways. EMBO J 2003, 22:4212-4222.

38. Freund A, Orjalo AV, Desprez PY, Campisi J: Inflammatory networks during cellular senescence: causes and consequences. Trends Mol Med 2010, 16:238-246

39. Rodier F, Campisi J: Four faces of cellular senescence. J Cell Biol 2011 , 192:547-556.

40. Zeng M, Smith AJ, Wietgrefe SW, Southern PJ, Schacker TW, Reilly CS, Estes JD, Burton GF, Silvestri G, Lifson JD, Carlis JV, Haase AT: Cumulative mechanisms of lymphoid tissue fibrosis and T cell depletion in HIV-1 and SIV infections. J Clin Invest 2011, 121:998-1008.

41. Schacker TW, Reilly C, Beilman GJ, Taylor J, Skarda D, Krason D, Larson M, Haase AT: Amount of lymphatic tissue fibrosis in HIV infection predicts magnitude of HAART-associated change in peripheral CD4 cell count. AIDS 2005, 19:2169-2171.

42. Chujo S, Shirasaki F, Kawara S, Inagaki Y, Kinbara T, Inaoki M, Takigawa M, Takehara K: Connective tissue growth factor causes persistent proalpha2 (I) collagen gene expression induced by transforming growth factor-beta in a mouse fibrosis model. J Cell Physiol 2005, 203:447-456.

43. Arnott JA, Nuglozeh E, Rico MC, Arango-Hisijara I, Odgren PR, Safadi FF, Popoff SN: Connective tissue growth factor (CTGF/CCN2) is a downstream mediator for TGF-beta1-induced extracellular matrix production in osteoblasts. J Cell Physiol 2007, 210:843-852.

44. Close GL, Kayani A, Vasilaki A, McArdle A: Skeletal muscle damage with exercise and aging. Sports Med 2005, 35:413-427.

45. Lexell J, Taylor CC, Sjostrom M: What is the cause of the ageing atrophy? Total number, size and proportion of different fiber types studied in whole vastus lateralis muscle from 15- to 83-year-old men. J Neurol Sci 1988, 84:275-294.

46. Porter MM, Vandervoort AA, Lexell J: Aging of human muscle: structure, function and adaptability. Scand J Med Sci Sports 1995, 5:129-142.

47. Effros RB, Fletcher CV, Gebo K, Halter JB, Hazzard WR, Horne FM, Huebner RE, Janoff EN, Justice AC, Kuritzkes D, Nayfield SG, Plaeger SF, Schmader KE,
Ashworth JR, Campanelli C, Clayton CP, Rada B, Woolard NF, High KP: Aging and infectious diseases: workshop on HIV infection and aging: what is known and future research directions. Clin Infect Dis 2008, 47:542-553.

48. Walston J, Hadley EC, Ferrucci L, Guralnik JM, Newman AB, Studenski SA Ershler WB, Harris T, Fried LP: Research agenda for frailty in older adults: toward a better understanding of physiology and etiology: summary from the American Geriatrics Society/National Institute on Aging Research Conference on frailty in older adults. J Am Geriatr Soc 2006, 54:991-1001.

49. Ruiz M, Cefalu C: Characteristics of frail patients in a geriatric-HIV program: the experience of an urban academic center at one year follow-up. J Int Assoc Physicians AIDS Care 2011, 10:138-143.

50. Fried LP, Tangen CM, Walston J, Newman AB, Hirsch C, Gottdiener J, Seeman T, Tracy R, Kop WJ, Burke G, McBurnie MA, Cardiovascular Health Study Collaborative Research Group: Frailty in older adults: evidence for a phenotype. J Gerontol A Biol Sci Med Sci 2001, 56:M146-M156.

51. Barbieri M, Ferrucci L, Ragno E, Corsi A, Bandinelli S, Bonafe M, Olivieri F, Giovagnetti S, Franceschi C, Guralnik JM, Paolisso G: Chronic inflammation and the effect of IGF-I on muscle strength and power in older persons. Am J Physiol Endocrinol Metab 2003, 284:E481-E487.

52. Krabbe KS, Pedersen M, Bruunsgaard $\mathrm{H}$ : Inflammatory mediators in the elderly. Exp Gerontol 2004, 39:687-699.

53. Conboy IM, Conboy MJ, Wagers AJ, Girma ER, Weissman IL, Rando TA: Rejuvenation of aged progenitor cells by exposure to a young systemic environment. Nature 2005, 433:760-764.

54. Estes JD: Role of collagen deposition in lymphatic tissues and immune reconstruction during HIV-1 and SIV infections. Curr HIV/AIDS Rep 2009, 6:29-35.

55. Estes JD, Haase AT, Schacker TW: The role of collagen deposition in depleting CD4+ T cells and limiting reconstitution in HIV-1 and SIV infections through damage to the secondary lymphoid organ niche. Semin Immunol 2008, 20:181-186.

56. Assmann EM, Alborghetti MR, Camargo ME, Kobarg J: FEZ1 dimerization and interaction with transcription regulatory proteins involves its coiledcoil region. J Biol Chem 2006, 281:9869-9881.

57. Hardy RR, Hayakawa K: Development of B cells producing natural autoantibodies to thymocytes and senescent erythrocytes. Springer Semin Immunopathol 2005, 26:363-375.

58. Katoh M: Identification and characterization of human DAAM2 gene in silico. Int J Oncol 2003, 22:915-920.

59. Gourlay CW, Carpp LN, Timpson P, Winder SJ, Ayscough KR: A role for the actin cytoskeleton in cell death and aging in yeast. J Cell Biol 2004, 164:803-809.

60. Kato JY, Yoneda-Kato N: Mammalian COP9 signalosome. Genes Cells 2009, 14:1209-1225.

61. Dahl HH, Brown GK, Brown RM, Hansen LL, Kerr DS, Wexler ID, Patel MS, De Meirleir L, Lissens W, Chun K, et al: Mutations and polymorphisms in the pyruvate dehydrogenase E1 alpha gene. Hum Mutat 1992, 1:97-102.

62. Kagi JH: Overview of metallothionein. Methods Enzymol 1991, 205:613-626.

63. Bober E, Buchberger-Seidl A, Braun T, Singh S, Goedde HW, Arnold HH: Identification of three developmentally controlled isoforms of human myosin heavy chains. Eur J Biochem 1990, 189:55-65.

64. Nystrom J, Hultenby K, Ek S, Sjolund J, Axelson H, Jirstrom K, Saleem MA, Nilsson K, Johansson ME: CRIM1 is localized to the podocyte filtration slit diaphragm of the adult human kidney. Nephrol Dial Transplant 2009, 24:2038-2044

65. Coppe JP, Patil CK, Rodier F, Krtolica A, Beausejour CM, Parrinello S, Hodgson JG, Chin K, Desprez PY, Campisi J: A human-like senescenceassociated secretory phenotype is conserved in mouse cells dependent on physiological oxygen. PLOS One 2010, 12;5:e9188.

66. Young AR, Narita M: SASP reflects senescence. EMBO Rep 2009, 10:228-230.

67. Bruunsgaard H, Andersen-Ranberg K, Jeune B, Pedersen AN, Skinhoj P, Pedersen BK: A high plasma concentration of TNF-alpha is associated with dementia in centenarians. J Gerontol A Biol Sci Med Sci 1999, 54: M357-M364.

68. Bruunsgaard H, Pedersen M, Pedersen BK: Aging and proinflammatory cytokines. Curr Opin Hematol 2001, 8:131-136.

69. De Martinis M, Franceschi C, Monti D, Ginaldi L: Inflamm-ageing and lifelong antigenic load as major determinants of ageing rate and longevity. FEBS Lett 2005, 579:2035-2039. 
70. Gopinath SD, Rando TA: Stem cell review series: aging of the skeletal muscle stem cell niche. Aging Cell 2008, 7:590-598.

71. Appay V, Rowland-Jones SL: Premature ageing of the immune system: the cause of AIDS? Trends Immunol 2002, 23:580-585.

72. Margolick JB, Chopra RK: Relationship between the immune system and frailty: pathogenesis of immune deficiency in HIV infection and aging. Aging 1992, 4:255-257.

73. Abad LW, Schmitz HR, Parker R, Roubenoff R: Cytokine responses differ by compartment and wasting status in patients with HIV infection and healthy controls. Cytokine 2002, 18:286-293.

74. Baracos V, Rodemann HP, Dinarello CA, Goldberg AL: Stimulation of muscle protein degradation and prostaglandin E2 release by leukocytic pyrogen (interleukin-1). A mechanism for the increased degradation of muscle proteins during fever. N Engl J Med 1983, 308:553-558.

75. Clowes GH Jr, George BC, Jr Villee CA, Saravis CA: Muscle proteolysis induced by a circulating peptide in patients with sepsis or trauma. $\mathrm{N}$ Engl J Med 1983, 308:545-552.

76. Thea DM, Porat R, Nagimbi K, Baangi M, St Louis ME, Kaplan G, Dinarello CA, Keusch GT: Plasma cytokines, cytokine antagonists, and disease progression in African women infected with HIV-1. Ann Intern Med 1996, 124:757-762.

77. Zoico $E$, Roubenoff $R$ : The role of cytokines in regulating protein metabolism and muscle function. Nutr Rev 2002, 60:39-51.

78. Tracey KJ, Wei H, Manogue KR, Fong Y, Hesse DG, Nguyen HT, Kuo GC, Beutler B, Cotran RS, Cerami A, et al: Cachectin/tumor necrosis factor induces cachexia, anemia, and inflammation. J Exp Med 1988, 167:12111227.

79. Cao PR, Kim HJ, Lecker SH: Ubiquitin-protein ligases in muscle wasting. Int J Biochem Cell Biol 2005, 37:2088-2097.

80. Lecker SH, Jagoe RT, Gilbert A, Gomes M, Baracos V, Bailey J, Price SR, Mitch WE, Goldberg AL: Multiple types of skeletal muscle atrophy involve a common program of changes in gene expression. FASEB J 2004, 18:3951.

81. Sandri M, Sandri C, Gilbert A, Skurk C, Calabria E, Picard A, Walsh K, Schiaffino S, Lecker SH, Goldberg AL: Foxo transcription factors induce the atrophy-related ubiquitin ligase atrogin-1 and cause skeletal muscle atrophy. Cell 2004, 117:399-412.

82. Bodine SC, Latres E, Baumhueter S, Lai VK, Nunez L, Clarke BA, Poueymirou WT, Panaro FJ, Na E, Dharmarajan K, Pan ZQ, Valenzuela DM, DeChiara TM, Stitt TN, Yancopoulos GD, Glass DJ: Identification of ubiquitin ligases required for skeletal muscle atrophy. Science 2001, 294:1704-1708.

83. Glass DJ: Skeletal muscle hypertrophy and atrophy signaling pathways. Int J Biochem Cell Biol 2005, 37:1974-1984.

84. Cai D, Frantz JD, Tawa NE Jr, Melendez PA, Oh BC, Lidov HG, Hasselgren PO, Frontera WR, Lee J, Glass DJ, Shoelson SE: IKKbeta/NF-kappaB activation causes severe muscle wasting in mice. Cell 2004, 119:285-298.

doi:10.1186/2044-5040-2-10

Cite this article as: Kusko et al.: Premature expression of a muscle fibrosis axis in chronic HIV infection. Skeletal Muscle 2012 2:10.

\section{Submit your next manuscript to BioMed Central and take full advantage of:}

- Convenient online submission

- Thorough peer review

- No space constraints or color figure charges

- Immediate publication on acceptance

- Inclusion in PubMed, CAS, Scopus and Google Scholar

- Research which is freely available for redistribution

Submit your manuscript at www.biomedcentral.com/submit
( Biomed Central 